\title{
Transcriptomics analysis reveals the high biodegradation efficiency of white- rot fungus Phanerochaete sordida YK-624 on native lignin
}

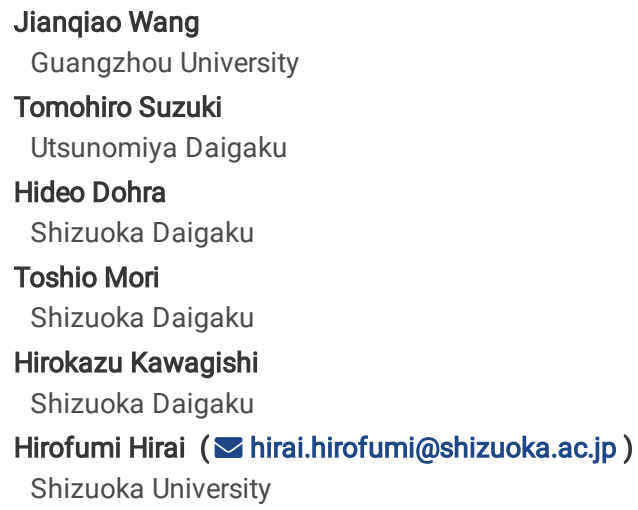

Research

Keywords: Phanerochaete sordida YK-624, lignin degradation, white-rot fungi, RNA-Seq

Posted Date: January 9th, 2020

DOI: https://doi.org/10.21203/rs.2.20480/v1

License: () (7) This work is licensed under a Creative Commons Attribution 4.0 International License. Read Full License

Version of Record: A version of this preprint was published at Journal of Bioscience and Bioengineering on June 1st, 2021. See the published version at https://doi.org/10.1016/j.jbiosc.2021.05.009. 


\section{Abstract}

Background

Lignocellulosic biomass is an organic matrix composed of cellulose, hemicellulose, and lignin. In nature, lignin degradation by basidiomycetes is the key step in lignocellulose decay. The white-rot fungus Phanerochaete sordida YK-624 (YK-624) has been extensively studied due to its high lignin degradation ability. In our previous study, it was demonstrated that YK-624 can secrete lignin peroxidase and manganese peroxidase for lignin degradation. However, the underlying mechanism for lignin degradation by YK-624 remains unknown.

Results

Here, we analyzed YK-624 gene expression following growth under ligninolytic and nonligninolytic conditions and compared the differentially expressed genes in YK-624 to those in the model white-rot fungus $P$. chrysosporium by next-generation sequencing. More ligninolytic enzymes and lignin-degrading auxiliary enzymes were upregulated in YK-624. This might explain the high degradation efficiency of YK-624. In addition, the genes involved in energy metabolism pathways, such as the TCA cycle, oxidative phosphorylation, lipid metabolism, carbon metabolism and glycolysis, were upregulated under ligninolytic conditions in YK-624.

Conclusions

In the present study, the first differential gene expression analysis of YK-624 under ligninolytic and nonligninolytic conditions was reported. The results obtained in this study indicated that YK-624 produces more energy- and lignin-degrading enzymes for more efficient lignin biodegradation.

\section{Background}

To create renewable clean energy resources, one of the most feasible methods is the development of biorefineries to produce biofuels and renewable chemicals [1, 2]. Lignocellulosic biomass is the most abundant carbon-neutral source and composed primarily of lignin, cellulose, and hemicellulose. Due to the high abundance and low cost of lignocellulosic substrates, they are considered attractive feedstocks for second generation biofuel production. Furthermore, unlike first-generation biofuel feedstocks, they do not compete with food and are therefore widely used as waste or byproducts from agricultural industries and forestry [3]. However, lignocellulose recalcitrance presents the largest obstacle in the biotechnological conversion of biomass during degradation of the aromatic polymer lignin.

Lignin is the second most abundant biopolymer and a major component of lignocellulosic biomass, constituting 25\% of woody plant cell walls. Because of its nonwater soluble, heterogeneous and optically inactive nature, lignin is highly resistant to chemobiological degradation [4]. In nature, lignin degradation by basidiomycetes is the key step in lignocellulose decay. It is known that some microorganisms can degrade lignin, but only white-rot fungi can degrade it to carbon dioxide [5]. This biodegradation process is initiated by one-electron oxidation and is mediated by some ligninolytic enzymes, including lignin peroxidase (LiP), manganese peroxidase (MnP), laccase and versatile peroxidase (VP). LiP and MnP were first reported in Phanerochaete chrysosporium [6, 7]. $\mathrm{LiP}$ was first reported in P. chrysosporium and is able to attack lignin by oxidative cleavage of nonphenolic aromatic substrates [8]. MnP can oxidize Mn ${ }^{2+}$ to $\mathrm{Mn}^{3+}$ to degrade lignin via lipid peroxidation reactions [6]. VPs combine LiP and MnP and oxidize LiP substrates and Mn ${ }^{2+}[9]$. Laccases, which are widely distributed in plants, insects and fungi, are blue multicopper oxidases, and they can oxidize substituted phenols by reducing molecular oxygen to $\mathrm{H}_{2} \mathrm{O}$ [10, 11]. In addition, other extracellular enzymes involved in lignin degradation have been reported, including aryl-alcohol oxidase, glyoxal oxidase, and aryl-alcohol dehydrogenases [12]. YK-624 exhibited higher ligninolytic activity than the model white-rot fungi P. chrysosporium or Trametes versicolor [13]. We have previously demonstrated that YK-624 could secrete LiP and MnP for lignin degradation [14-16]. However, the underlying mechanism responsible for the lignin degradation of this white-rot fungus remains unknown.

Here, we report the first transcriptomic data of the hyper lignin-degrading white-rot fungus YK-624. In this paper, we aimed to investigate the key genes for lignin degradation and to elucidate the underlying mechanism of the high ligninolytic activity of YK-624. We analyzed YK-624 gene expression following growth under ligninolytic and nonligninolytic conditions and compared differential gene expression to P. chrysosporium by next-generation sequencing (RNASeq).

\section{Results And Discussion Lignin degradation}

The ligninolytic properties of the two strains were investigated. YK-624 showed $32.7 \%$ ligninolytic activity and 13.9\% weight loss (Fig. 1). P. chrysosporium showed only $6.7 \%$ ligninolytic activity and $4.6 \%$ weight loss (Fig. 1). These results are in good agreement with those of our previous study, in which YK-624 had significantly higher ligninolytic activity [13].

\section{Sequence assembly}

Total RNA was extracted from biological triplicates of YK-624 and P. chrysosporium on wood meal and holocellulose meal after an incubation period of 10 days (termed YKwood, YKholo, PCwood, and PCholo). cDNA libraries were constructed after purification of mRNA for sequencing by MiSEq. Raw paired-end (2 $\times 76$ bp) sequences from mRNAs were obtained: 33,539,967 and 32,722,656 reads for YK-624 and P. chrysosporium, respectively. The high-quality reads (quality values $>30$ ) were assembled into 44,250 (N50 length = 1,020 bp) and 33,340 (N50 length = 1,426 bp) unigenes. Furthermore, ribosomal RNA (rRNA) 
and genes were obtained from mitochondrial sequences that matched entries in the SILVA (version 111) rRNA database [17] and were excluded from Trinity unigenes.

\section{Functional annotations}

The unigenes were searched against the Swiss-Prot and Uniref 90 databases by local BLASTX and annotated with gene ontology (GO) terms for functional annotation (Table S1; Table S2). Differentially expressed genes (DEGs) between the ligninolytic and nonligninolytic conditions were identified in this study. There were 985 upregulated genes and 1,085 downregulated genes in YK-624 (Table S1). In P. chrysosporium, 1,257 genes were upregulated, while 1,296 genes were downregulated (Table S2). The role of the ligninolytic enzymes LiP and MnP in lignin degradation has been extensively documented [18]. In the present study, three LiPs and five MnPs were upregulated in YK-624, while only two LiPs and one MnP were upregulated in P. chrysosporium under ligninolytic conditions (Tables S1 and S2). This may explain the high degradation efficiency of YK-624. In addition, some lignin-degrading auxiliary enzymes, including glyoxal oxidase (EC:1.2.3.5), glucose oxidase (EC:1.1.3.4), and aryl-alcohol dehydrogenase (EC:1.1.1.91), were also detected under ligninolytic conditions. Glyoxal oxidase and glucose oxidase are involved in the oxidative generation of $\mathrm{H}_{2} \mathrm{O}_{2}$ [19]. Aryl-alcohol dehydrogenase is produced during the ligninolytic growth phase of the fungus [20]. Moreover, ATPase, ATP synthase (EC:3.6.3.14) and NADH-cytochrome $b_{5}$ reductases were upregulated in YK-624, suggesting that it generated more energy than P. chrysosporium under ligninolytic conditions.

GO enrichment analysis of the two white-rot fungi was performed in the present study. We found 19 upregulated and 12 downregulated GO terms in YK-624. The most enriched GO terms were "ATPase activity" in the molecular functions category, "transport" and "transmembrane transport" in the biological processes category, and "ribosome" in the cellular components category under ligninolytic conditions (Table 1). These results further demonstrated that YK624 produced more energy for lignin degradation. In contrast, only "hydrogen ion transmembrane transporter activity" in molecular functions and "ATP synthesis coupled proton transport" in biological processes were significantly enriched in P. chrysosporium (Table S3). It was reported that the transportation of lignin-derived aromatic molecules is very important for biomass applications [21]. This transportation activity was detected in both fungi. 
Table 1

Enrichment of gene ontology terms in differentially expressed sequences in P. sordida YK-624 detected by PAGE

\begin{tabular}{|c|c|c|c|c|c|c|}
\hline \multicolumn{2}{|c|}{ GO_name } & \multirow{2}{*}{$\begin{array}{l}\text { Go_id } \\
\text { GO:0006810 }\end{array}$} & \multirow{2}{*}{$\begin{array}{l}\text { Number of sequences } \\
128\end{array}$} & \multirow{2}{*}{$\begin{array}{l}Z \text { core } \\
6.478\end{array}$} & \multirow{2}{*}{$\begin{array}{l}\text { P-value } \\
9.32 \mathrm{E}-11\end{array}$} & \multirow{2}{*}{$\begin{array}{l}\text { FDR } \\
\text { 4.02E-09 }\end{array}$} \\
\hline $\mathrm{BP}$ & transport & & & & & \\
\hline $\mathrm{BP}$ & transmembrane transport & G0:0055085 & 407 & 5.933 & 2.98E-09 & 1.07E-07 \\
\hline MF & ATPase activity, coupled to transmembrane movement of substances & G0:0042626 & 57 & 5.326 & $1.01 \mathrm{E}-07$ & 3.1E-06 \\
\hline MF & metal ion transmembrane transporter activity & G0:0046873 & 16 & 5.011 & $5.42 \mathrm{E}-07$ & 1.37E-05 \\
\hline $\mathrm{BP}$ & metal ion transport & GO:0030001 & 22 & 4.128 & 3.67E-05 & 0.0007 \\
\hline $\mathrm{CC}$ & ribosome & G0:0005840 & 110 & 3.822 & 0.0001 & 0.0019 \\
\hline MF & structural constituent of ribosome & G0:0003735 & 112 & 3.782 & 0.0002 & 0.0021 \\
\hline $\mathrm{BP}$ & glycolytic process & G0:0006096 & 12 & 3.685 & 0.0002 & 0.0029 \\
\hline $\mathrm{BP}$ & translation & G0:0006412 & 109 & 3.576 & 0.0003 & 0.0042 \\
\hline $\mathrm{CC}$ & membrane & GO:0016020 & 276 & 3.336 & 0.0008 & 0.0097 \\
\hline MF & ATPase activity & GO:0016887 & 106 & 3.158 & 0.0016 & 0.0171 \\
\hline $\mathrm{BP}$ & DNA replication & GO:0006260 & 44 & 3.123 & 0.0018 & 0.0184 \\
\hline $\mathrm{BP}$ & response to oxidative stress & GO:0006979 & 21 & 3.039 & 0.0024 & 0.0223 \\
\hline MF & serine-type endopeptidase activity & GO:0004252 & 17 & 3.001 & 0.0027 & 0.0242 \\
\hline MF & transporter activity & GO:0005215 & 21 & 2.930 & 0.0034 & 0.0293 \\
\hline MF & threonine-type endopeptidase activity & G0:0004298 & 16 & 2.848 & 0.0044 & 0.0339 \\
\hline $\mathrm{CC}$ & proteasome core complex & G0:0005839 & 16 & 2.848 & 0.0044 & 0.0339 \\
\hline $\mathrm{BP}$ & proteolysis involved in cellular protein catabolic process & GO:0051603 & 16 & 2.848 & 0.0044 & 0.0339 \\
\hline MF & transferase activity, transferring acyl groups & Go:0016746 & 25 & 2.737 & 0.0062 & 0.0432 \\
\hline MF & cation binding & GO:0043169 & 14 & -2.768 & 0.0056 & 0.0406 \\
\hline MF & nucleic acid binding & Go:0003676 & 218 & -2.781 & 0.0054 & 0.0404 \\
\hline MF & nutrient reservoir activity & GO:0045735 & 11 & -3.062 & 0.0022 & 0.0216 \\
\hline MF & protein kinase activity & GO:0004672 & 260 & -3.961 & 7.46E-05 & 0.0012 \\
\hline $\mathrm{BP}$ & protein phosphorylation & G0:0006468 & 263 & -3.998 & $6.39 \mathrm{E}-05$ & 0.0011 \\
\hline MF & structural constituent of cell wall & GO:0005199 & 13 & -4.293 & $1.76 \mathrm{E}-05$ & 0.0003 \\
\hline $\mathrm{CC}$ & fungal-type cell wall & GO:0009277 & 13 & -4.293 & $1.76 \mathrm{E}-05$ & 0.0003 \\
\hline MF & protein binding & GO:0005515 & 990 & -5.000 & 5.73E-07 & 1.37E-05 \\
\hline BP & carbohydrate metabolic process & G0:0005975 & 216 & -8.000 & 1.33E-15 & 7.19E-14 \\
\hline MF & hydrolase activity, hydrolyzing O-glycosyl compounds & G0:0004553 & 155 & -8.027 & $8.88 \mathrm{E}-16$ & 6.39E-14 \\
\hline $\mathrm{CC}$ & extracellular region & GO:0005576 & 39 & -10.671 & 0 & 0 \\
\hline MF & cellulose binding & GO:0030248 & 33 & -10.816 & 0 & 0 \\
\hline
\end{tabular}


Table 2

Enrichment of gene ontology terms in differentially expressed sequences in P. chrysosporium detected by PAGE

\begin{tabular}{|c|c|c|c|c|c|c|}
\hline \multicolumn{2}{|c|}{ GO_name } & \multirow{2}{*}{$\begin{array}{l}\text { Go_id } \\
\text { GO:0015078 }\end{array}$} & \multirow{2}{*}{$\begin{array}{l}\text { Number of sequences } \\
13\end{array}$} & \multirow{2}{*}{$\begin{array}{l}Z \text { core } \\
5.096\end{array}$} & \multirow{2}{*}{$\begin{array}{l}\text { P-value } \\
\text { 3.47E-07 }\end{array}$} & \multirow{2}{*}{$\begin{array}{l}\text { FDR } \\
\text { 1.43E-05 }\end{array}$} \\
\hline MF & hydrogen ion transmembrane transporter activity & & & & & \\
\hline $\mathrm{BP}$ & ATP synthesis coupled proton transport & GO:0015986 & 10 & 4.551 & 5.35E-06 & 0.0002 \\
\hline $\mathrm{BP}$ & carbohydrate metabolic process & G0:0005975 & 201 & -8.175 & $2.22 \mathrm{E}-16$ & $1.15 \mathrm{E}-14$ \\
\hline MF & hydrolase activity, hydrolyzing 0-glycosyl compounds & GO:0004553 & 139 & -10.242 & 0 & 0 \\
\hline $\mathrm{CC}$ & extracellular region & GO:0005576 & 34 & -15.308 & 0 & 0 \\
\hline MF & cellulose binding & G0:0030248 & 28 & -17.335 & 0 & 0 \\
\hline
\end{tabular}

To explore the underlying mechanisms for the high lignin degradation of YK-624, we mapped all upregulated genes in YK-624 and P. chrysosporium to Kyoto Encyclopedia of Genes and Genomes (KEGG) metabolic pathways. Our results suggested that the biosynthesis of secondary metabolites and their metabolic pathways were the most frequently represented pathways. We compared the DEGs in YK-624 and P. chrysosporium under ligninolytic conditions according to the KEGG pathway assignments. A summary of transcripts involved in the lignin-degrading pathways of YK-624 is shown (Fig. 2) and the pathways include carbon metabolism, the tricarboxylic acid cycle (TCA cycle), oxidative phosphorylation and xenobiotic biodegradation. In all, $18 \mathrm{genes}$ were mapped to these pathways in YK-624, but only 4 were mapped to these pathways in P. chrysosporium. The TCA cycle and oxidative phosphorylation pathways involve important biochemical reactions in energy production. In YK-624, citrate synthase (EC:2.3.3.1), aconitate hydratase (EC:4.2.1.3), isocitrate lyase (EC:4.1.3.1), ubiquinol-cytochrome $\mathrm{c}$ reductase cytochrome b subunit, cytochrome $\mathrm{c}$ oxidase subunit 1 (EC:1.9.3.1) and F-type $\mathrm{H}^{+}$-transporting ATPase subunit c, which are involved in these two processes, were upregulated (Fig. 2 and Table S1). At the same time, pyruvate dehydrogenase E1 component beta subunit (EC:1.2.4.1) and acetyl-CoA synthetase (EC:6.2.1.1) were also upregulated, and the products of the metabolic pathways that they are involved in will go further into the TCA cycle. The other 4 upregulated genes were malonate-semialdehyde dehydrogenase (acetylating)/methylmalonate-semialdehyde dehydrogenase (EC:1.2.1.18 1.2.1.27), fructose-1,6-bisphosphatase I (EC:3.1.3.11), glycine hydroxymethyltransferase (EC:2.1.2.1) and catalase (EC:1.11.1.6), which are responsible for lipid metabolism, glycolysis, and carbon metabolism; thus, YK-624 generated more ATP for lignin degradation. All the energy generated by accelerating metabolic pathways as described above will be used for xenobiotic biodegradation. Aldehyde dehydrogenase (NAD $\left.{ }^{+}\right)(E C: 1.2 .1 .3)$, salicylate hydroxylase (EC:1.14.13.1), carboxymethylenebutenolidase (EC:3.1.1.45), amidase (EC:3.5.1.4), nitrilase (EC:3.5.5.1) and phenol 2-monooxygenase (EC:1.14.13.7), which are involved in xenobiotic biodegradation, were also upregulated in YK-624 (Fig. 2). It was reported that the addition of alkali lignin (Sigma 45-471003) can increase energy production in the bacterium Enterobacter lignolyticus SCF1 [22]. ATP-dependent mechanisms play an important role in aromatic compounds derived from lignin degradation [21]. Hereby, we assume that YK-624 produces more energy to degrade lignin.

\section{Conclusions}

Here, we report the first transcriptomic information based on next-generation sequencing technology of YK-624. We investigated the genes involved in lignin degradation by comparing them to those of P. chrysosporium. This work shows that more ligninolytic enzymes (LiPs and MnPs) are upregulated in YK-624 under ligninolytic conditions. Furthermore, the genes related to energy production that accelerate metabolic pathways such as the TCA cycle, oxidative phosphorylation, lipid metabolism, carbon metabolism, and glycolysis are upregulated in YK-624. All the energy generated might be used for degrading lignin. Therefore, YK-624 can biodegrade lignin more efficiently. This study may provide new insights into genetic engineering for lignin biodegradation in the future.

\section{Methods Decay test}

Wise method was used in this study for removing lignin from beech wood meal [23], in which a sodium chlorite/acetic acid mixture was used at $70 \sim 80{ }^{\circ} \mathrm{C}$ in a water bath, and sodium chlorite and acetic acid were added every $1 \mathrm{~h}$ for up to $8 \mathrm{~h}$. After the reaction, the slurry was filtered, and the solids were washed with cold distilled water and acetone. The solids were used as holocellulose medium (the Klason lignin was below 1\%) after air-drying.

After YK-624 and P. chrysosporium were incubated on PDA plates at $30^{\circ} \mathrm{C}$ for 3 days, the growing edge of the 10-mm-diameter mycelium disks was punched out. One disk was inoculated into $0.5 \mathrm{~g}$ extractive-free beech wood meal (ligninolytic) or holocellulose meal (nonligninolytic) (60 $80 \mathrm{mesh}$ ) and $1.25 \mathrm{~mL}$ Kirk medium (consisting of Kirk salt solution and 2,2-dimethylsuccinic acid) as described by Tien and Kirk [7]. The weight loss and lignin content of each condition were determined after 10 days of incubation using our previous method [13].

\section{RNA extraction, cDNA library preparation and sequencing}

Based on the incubation method described as the decay test, total RNA was extracted from biological triplicates of YK-624 and P. chrysosporium on $1 \mathrm{~g}$ wood and holocellulose media. After 10 days of incubation, each sample was ground into a fine powder in liquid nitrogen. Extraction of total RNA was conducted using Concert Plant RNA Reagent (Invitrogen, United States) and further purified with the Qiagen RNeasy Mini Kit (Hilden, Germany). RNA quantity and quality

Page 5/9 
were determined based on the method described by Garg et al., 2010 [24]. RNA quality was assessed by agarose gel electrophoresis and the OD260/OD280 ratio.

The subsequent cDNA library construction method was described in our previous study [25]. Briefly, total RNA samples were treated and purified with a DNase I and Qiagen RNeasy Mini Kit, respectively. First-strand cDNA was synthesized using an oligo-dT primer and PrimeScript reverse transcriptase (Takara). Then, libraries for strand-specific RNA sequencing were performed in two individual sequencing runs. Paired-end sequences of $75 \mathrm{bp}$ were obtained using an Illumina MiSeq system.

\section{Transcriptome assembly and functional annotation}

RNA-Seq and differential gene expression analyses were performed as previously described [25]. The paired-end reads were assembled by Trinity [26] after quality trimming of the adapter sequences using cutadapt ver. 1.8.1. The detailed transcriptome assembly and DEG identification methods were described previously. The P-value was determined by the false discovery rate (FDR). DEGs were set at $>1$-fold up- or downregulated (FDR $<0.05)$ in this study.

Functional annotation of the unigenes was performed in the Swiss-Prot and Uniref 90 databases by a local BLASTX algorithm [27]. KEGG pathways and GO annotation were performed and assigned to unigenes using the KEGG automatic annotation server (KAAS) and InterproScan [28, 29].

\section{Abbreviations}

ACO, aconitate hydratase; ACSS, acetyl-CoA synthetase; ALDH, aldehyde dehydrogenase (NAD+); ATPeFOC, F-type H+-transporting ATPase subunit c; CAHY, carboxymethylenebutenolidase; COX1, cytochrome $c$ oxidase subunit $1 ; C S$, citrate synthase; CYTB, ubiquinol-cytochrome $c$ reductase cytochrome b subunit; DEGs, differentially expressed genes; FBP, fructose-1,6-bisphosphatase l; glyA, glycine hydroxymethyltransferase; GO, gene ontology; ICL, isocitrate lyase; katE, catalase; KEGG, Kyoto Encyclopedia of Genes and Genomes; LiP, lignin peroxidase; mmsA, malonate-semialdehyde dehydrogenase (acetylating) / methylmalonate-semialdehyde dehydrogenase; MnP, manganese peroxidase; PDHB, pyruvate dehydrogenase E1 component beta subunit; PHMO, phenol 2monooxygenase; SALH, salicylate hydroxylase; VP, versatile peroxidase

\section{Declarations}

\section{Acknowledgements}

The authors acknowledged the financial support by the Japan Society for the Promotion of Science.

\section{Ethical approval and consent to participate}

Not applicable.

\section{Consent for publication}

All the authors consent to publish this research work.

\section{Availability of supporting data}

The data sets supporting the results of this article are available in the DDBJ Sequence Read Archive (DRA) (accession numbers for the sequences of $P$. sordida YK-624 and P. chrysosporium are DRA009154 and DRA009155, respectively).

\section{Competing interests}

The authors declare that they have no competing interests.

\section{Funding}

This research was supported by a JSPS International Research Fellow (Faculty of Agriculture, Shizuoka University; ID No.: 15F15100).

\section{Authors' contributions}

$\mathrm{HH}$ conceived and designed all experiments. JW and TS performed all experiments. HH, JW, TS and HD performed data analysis. TM and HK gave advice on the experiments. JW, TS and $\mathrm{HH}$ wrote the manuscript. All authors commented and approved the final manuscript.

\section{References}

1. Maurya DP, Singla A, Negi S. An overview of key pretreatment processes for biological conversion of lignocellulosic biomass to bioethanol. 3 Biotech. 2015;5:597-605.

2. Ragauskas AJ, Beckham GT, Biddy MJ, Chandra R, Chen F, Davis MF, Davison BH, Dixon RA, Gilna P, Keller M, Langan P, Naskar AK, Saddler JN, Tschaplinski TJ, Tuskan GA, Wyman CE. Lignin Valorization: Improving Lignin Processing in the Biorefinery. Science. 2014;344:1246843.

3. Nonhebel Renewable energy and food supply: will there be enough land? Renew. Sustain. Energy Rev. 2005;9:191-201. 
4. Sánchez C. Lignocellulosic residues: biodegradation and bioconversion by fungi. Adv. 2009;27(2):185-194.

5. Bumpus JA, Tien M, Wright D, Aust SD. Oxidation of persistent environmental pollutants by a white rot fungus. Science.1985;228:1434-1436.

6. Glenn JK, Gold MH. Purification and characterization of an extracellular Mn(II)-dependent peroxidase from the lignin-degrading basidiomycete Phanerochaete chrysosporium. Arch. Biochem. Biophys. 1985; 242:329-341.

7. Tien M, Kirk Lignin peroxidase of Phanerochaete chrysosporium. Method Enzymol. 1988;161:238-249.

8. Schoemaker HE, Lundell TK, Floris R, Glumoff T, Winterhalter KH, Piontek K. Do carbohydrates play a role in the lignin peroxidase cycle? Redox catalysis in the endergonic region of the driving force. Bioorg. Med. Chem. 1994;2(6):509-519.

9. Camarero S, Sarkar S, Ruiz-Dueñas FJ, Martínez MJ, Martínez AT. Description of a versatile peroxidase involved in the natural degradation of lignin that has both manganese peroxidase and lignin peroxidase substrate interaction sites. J. Biol. Chem. 1999;274(15):10324-10330.

10. Mayer AM, Staples RC. Laccase: new functions for an old enzyme. Phytochemistry. 2002;60:551-565.

11. Wong DW. Structure and action mechanism of ligninolytic enzymes. Appl. Biochem. Biotechnol. 2009;157(2):174-209.

12. Martínez AT, Speranza M, Ruiz-Dueñas FJ, Ferreira P, Camarero S, Guillén F, Martínez MJ, Gutiérrez A, del Río JC. Biodegradation of lignocellulosics: microbial, chemical, and enzymatic aspects of the fungal attack of lignin. Int. Microbiol. 2005;8(3):195-204.

13. Hirai H, Kondo R, Sakai K. Screening of lignin degrading fungi and their ligninolytic enzyme activities during biological bleaching of kraft pulp. Mokzai Gakkaishi. 1994;40:980-986.

14. Hirai H, Kondo R, Sakai K. Effect of metal ions on biological bleaching of kraft pulp with Phanerochaete sordida YK-624. Mokuzai Gakkaishi. 1995;41:6975.

15. Sugiura M, Hirai H, Nishida T. Purification and characterization of a novel lignin peroxidase from white-rot fungus Phanerochaete sordida YK-624. FEMS Microbiol. Lett. 2003;224:285-290.

16. Hirai H, Sugiura M, Kawai S, Nishida T. Characteristics of novel lignin peroxidases produced by white-rot fungus Phanerochaete sordida YK-624. FEMS Microbiol. Lett. 2005;246:19-24.

17. Pruesse E, Quast C, Knittel K, Fuchs BM, Ludwig W, Peplies J, Glöckner FO. SILVA: a comprehensive online resource for quality checked and aligned ribosomal RNA sequence data compatible with ARB. Nucleic Acids Res. 2007;35:7188-7196.

18. Hatakka A. Lignin-modifying enzymes from selected white-rot fungi: production and role in lignin degradation. FEMS Microbiol. Rev. 1994;13:125-135.

19. Janusz G, Pawlik A, Sulej J, Swiderska-Burek U, Jarosz-Wilkolazka A, Paszczynski A. Lignin degradation: microorganisms, enzymes involved, genomes analysis and evolution. FEMS Microbiol. Rev. 2017;41(6):941-962.

20. Muheim A, Waldner R, Sanglard D, Reiser J, Schoemaker HE, Leisola MS. Purification and properties of an aryl-alcohol dehydrogenase from the white-rot fungus Phanerochaete chrysosporium. Eur. J. Biochem. 1991;195(2):369-375.

21. Michalska K, Chang C, Mack JC, Zerbs S, Joachimiak A, Collart FR. Characterization of transport proteins for aromatic compounds derived from lignin: benzoate derivative binding proteins. J. Mol. Biol. 2012;423(4):555-575.

22. Deangelis KM, Sharma D, Varney R, Simmons B, Isern NG, Markilllie LM, Nicora C, Norbeck AD, Taylor RC, Aldrich JT, Robinson EW. Evidence supporting dissimilatory and assimilatory lignin degradation in Enterobacter lignolyticus Front Microbiol. 2013;4:280.

23. Wise LE, Murphy M, D'Addieco AA. Chlorite holocellulose, its fractionation and bearing on summative wood analysis and on studies on the hemicelluloses. Paper Trade. 1946;122(2):35-43.

24. Garg R, Sahoo A, Tyagi AK, Jain M. Validation of internal control genes for quantitative gene expression studies in chickpea. Biochem. Biophy. Res. Commun. 2010;396:283-8.

25. Wang J, Suzuki T, Dohra H, Takigami S, Kako H, Soga A, Kamei I, Mori T, Kawagishi H, Hirai H. Analysis of ethanol fermentation mechanism of ethanol producing white-rot fungus Phlebia MG-60 by RNA-seq. BMC Genomics. 2016;17(1):616.

26. Haas BJ, Papanicolaou A, Yassour M, Grabherr M, Blood PD, Bowden J, Couger MB, Eccles D, Li B, Lieber M, Macmanes MD, Ott M, Orvis J, Pochet N, Strozzi F, Weeks N, Westerman R, William T, Dewey CN, Henschel R, Leduc RD, Friedman N, Regev A. De novo transcript sequence reconstruction from RNA-seq using the Trinity platform for reference generation and analysis. Nat. Protoc. 2013;8(8):1494-1512.

27. Bairoch A, Boeckmann B. The SWISS-PROT protein sequence data bank. Nucleic Acids Res. 1991;19:2247-2249.

28. Kanehisa,M., and Goto,S. (2000) KEGG: kyoto encyclopedia of genes and genomes. Nucleic Acids Res., 28, 27-30.

29. Harris MA, Clark J, Ireland A, Lomax J, Ashburner M, Foulger R, Eilbeck K, Lewis S, Marshall B, Mungall C, Richter J, Rubin GM, Blake JA, Bult C, Dolan M, Drabkin H, Eppig JT, Hill DP, Ni L, Ringwald M, Balakrishnan R, Cherry JM, Christie KR, Costanzo MC, Dwight SS, Engel S, Fisk DG, Hirschman JE, Hong EL, Nash RS, Sethuraman A, Theesfeld CL, Botstein D, Dolinski K, Feierbach B, Berardini T, Mundodi S, Rhee SY, Apweiler R, Barrell D, Camon E, Dimmer E, Lee V, Chisholm R, Gaudet P, Kibbe W, Kishore R, Schwarz EM, Sternberg P, Gwinn M, Hannick L, Wortman J, Berriman M, Wood V, de la Cruz N, Tonellato P, Jaiswal P, Seigfried T, White R. The Gene Ontology (GO) database and informatics resource. Nucleic Acids Res. 2004;32:D258-261.

\section{Figures}




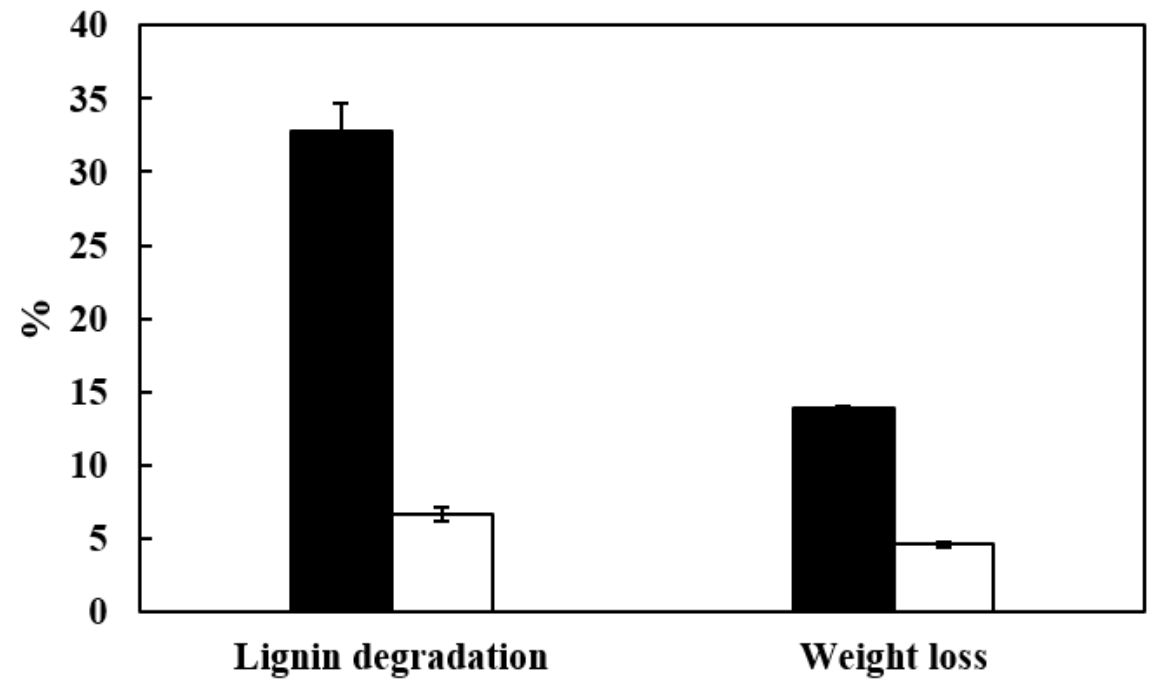

Figure 1

Ligninolytic properties of P. sordida YK-624 (black) and P. chrysosporium (white). The values are presented as the mean \pm standard deviation of triplicate samples.

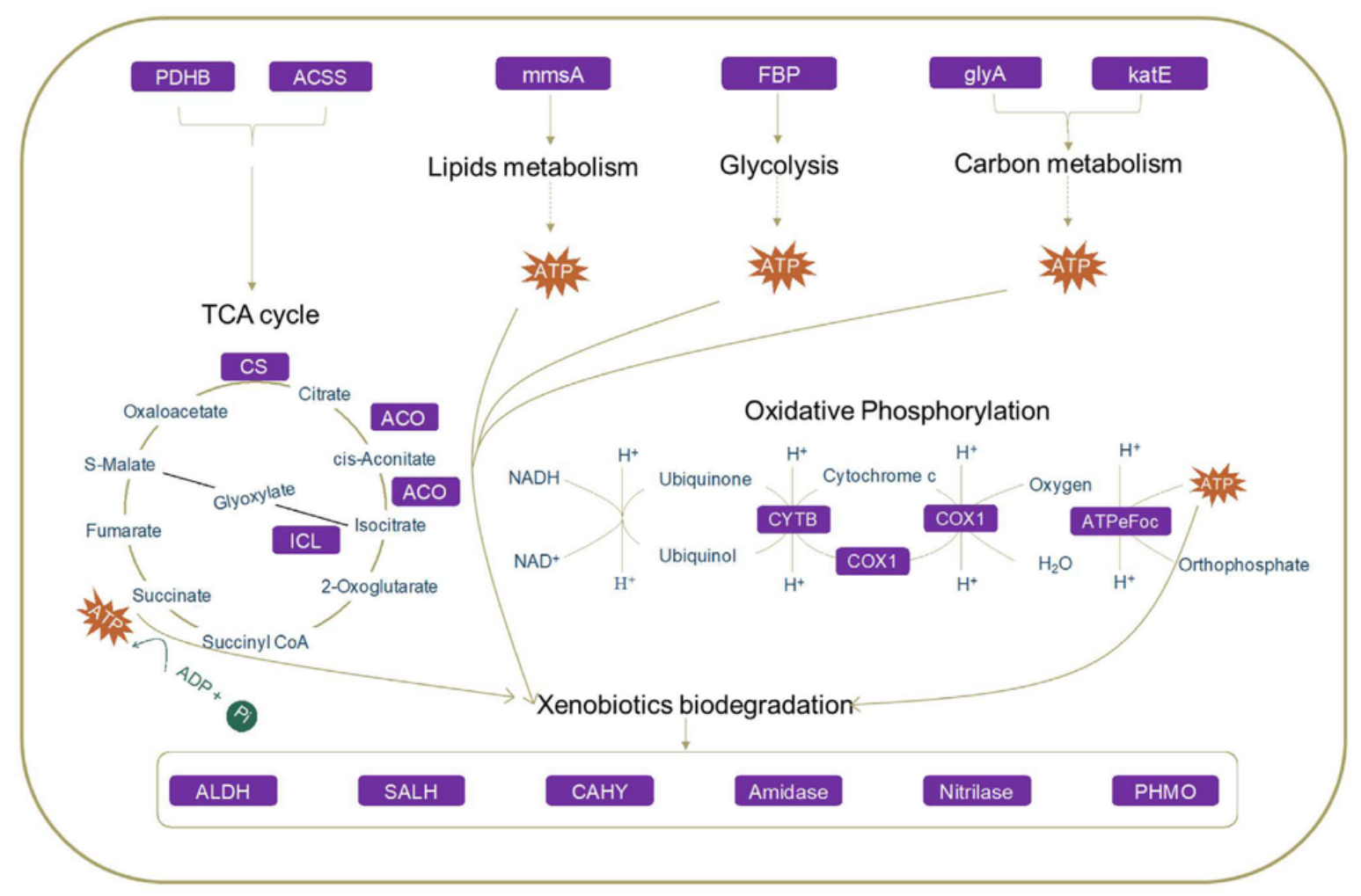

Figure 2

Summary of upregulated DEGs of P. sordida YK-624 under ligninolytic condition involved in lignin degradation. PDHB: pyruvate dehydrogenase E1 component beta subunit [EC:1.2.4.1]; ACSS: acetyl-CoA synthetase [EC:6.2.1.1]; mmsA: malonate-semialdehyde dehydrogenase (acetylating) / methylmalonate- 
semialdehyde dehydrogenase [EC:1.2.1.18 1.2.1.27]; FBP: fructose-1,6-bisphosphatase I [EC:3.1.3.11]; glyA: glycine hydroxymethyltransferase [EC:2.1.2.1]; katE: catalase [EC:1.11.1.6]; CS: citrate synthase [EC:2.3.3.1]; ACO: aconitate hydratase [EC:4.2.1.3]; ICL: isocitrate lyase [EC:4.1.3.1]; CYTB: ubiquinolcytochrome $\mathrm{c}$ reductase cytochrome b subunit; COX1: cytochrome c oxidase subunit 1 [EC:1.9.3.1]; ATPeF0C: F-type H+-transporting ATPase subunit c; ALDH: aldehyde dehydrogenase (NAD+) [EC:1.2.1.3]; SALH: salicylate hydroxylase [EC:1.14.13.1]; CAHY: carboxymethylenebutenolidase [EC:3.1.1.45]; PHMO: phenol 2-monooxygenase [EC:1.14.13.7].

\section{Supplementary Files}

This is a list of supplementary files associated with this preprint. Click to download.

- Tables1.xlsx

- Tables2.xlsx 